\title{
Single-Layer vs. Double-Layer Donor Scalp Wound Closure in Strip Harvest
}

\author{
Muhammad Ahmad \\ Plastic and Hair Restorative Surgeon, Hair \\ Transplant Institute, Islamabad, Pakistan
}

No potential conflict of interest relevant to this article was reported.
Background To know the difference between single and double-layer wound closure. Methods The study was conducted in ten patients undergoing first session of hair restoration surgery by 'strip method' under local anaesthesia. Informed consent was obtained from all the patients. The length and width of the strip were marked pre-operatively. Three points were marked, one in the midline ' $O$ ' and one on either side, ' $A$ ' \& ' $B$ ' at $8 \mathrm{~cm}$ from ' $O$ '. After removing the strip, five deep, interrupted, absorbable sutures were applied on left half but not on the right half. Lower edge trichophytic closure was performed with no undermining of the skin edges. A single continuous non-absorbable sutures was used to close the skin. The stitches were removed on the 10th postoperative day. The patients were assessed at 9 months interval postoperatively.

Results The mean age of the patients was 33.8 years. The average width of the strip removed was $15.1 \mathrm{~mm}$. The average width of the scar on single-layer closure was $1.57 \mathrm{~mm}$ and $1.58 \mathrm{~mm}$ on the double-layer closure. The mobility of the scar was $7.9 \mathrm{~mm}$ on single-layer closure and $6.2 \mathrm{~mm}$ on double-layer closure. There was no statistical significance in the scar size and mobility of single-layer vs double-layer closure. The time of surgery was more in double-layer closure and the extra cost of the suture material. Conclusions There was no difference in scar quality. The extra time and money was saved in single-layer closure. The mobility of the scar was also found to be better in single-layer closure.

Keywords Hair, Scalp, Surgery plastic, Suture techniques

\section{INTRODUCTION}

A surgical incision causes a scar which can lead to functional, cosmetic and psychologic problems. Scar tissue can be distinguished from normal skin by an abnormal colour, different thickness, decreased pliability, contraction or expansion of its surface area [1]. Multiple factors lead to typical features of a scar. These include location, size, aetiology, suturing techniques, suture material, wound

Received: Jun 1, 2016 Revised: Jan 11, 2017 Accepted: Jan 12, 2017 Correspondence: Muhammad Ahmad Plastic and Hair Restorative Surgeon, Hair Transplant Institute, House No. 1-B, Street 16, Main Kohistan Road, Sector F-8/3, Islamabad, Pakistan. E-mail: plasticsurgeonpk@yahoo.com

Copyright @ 2017 The Korean Society for Aesthetic Plastic Surgery.

This is an Open Access article distributed under the terms of the Creative Commons Attribution Non-Commercial License (http://creativecommons.org/licenses/by-nc/4.0/) which permits unrestricted non-commercial use, distribution, and reproduction in any medium, provided the original work is properly cited. www.e-aaps.org care, wound healing, and individual's race, age, and genetic predisposition [2]. The number of hair restoration surgeries is increasing every year [3]. The aesthetically performed hair restoration procedure has two components; the first is the natural angle and strategic distribution of hair in the recipient area and the second is the minimally visible scar resulting from the strip closure. Traditionally various methods of wound closure are mentioned which include single-layer closure, double-layer closure, the staples etc. [4-6]. Other factors like trichophytic closure (one-margin or double-margin) also help to minimize the scar width which is proportionate to the tension on the scar edges [4]. Although various suture materials have been compared but no study to date has been conducted in the same patient using single-layer (non-absorbable) and doublelayer (deep absorbable and superficial non-absorbable suture) closure [5-7].

The following study was conducted to know the difference between single and double-layer closure. 


\section{METHODS}

The study was conducted in ten patients undergoing first session of hair restoration surgery by 'strip method. The procedures were carried out under local anaesthesia. All the patients were thoroughly explained about the procedure and informed consent was obtained. The length and width of the strip were marked pre-operatively according to the plan. Tumescent anaesthesia was used.

\section{Surgical technique}

Three points were marked. The centre point (o) was marked in the midline. Two points (A and B) were marked on either side from point ' $\mathrm{O}$ ' at $8 \mathrm{~cm}$ (Fig. 1). These point (A and B) were used in postoperative assessment. The strip was harvested in prone position. Five deep, interrupted stitches at equal distance were applied using absorbable suture material on left half at subcutaneous level but not on the right half. No undermining of the skin edges was performed. Lower edge trichophytic closure (1-2 mm) was performed. First the scoring was done using the sharp end of surgical blade and then the skin was removed using scissors (Fig. 2). The continuous non-absorbable sutures were used to close the skin starting from left end of the wound and continuing towards the right end. Postoperative oral antibiotics (1st generation cephalosporins) were given for five days. The stitches were removed on the 10th postoperative day. The patients were asked to visit at 9-month interval. The parameters noted were width of the scar, the vertical mobility (measured on a millimeter scale), time spent in stitching the wound (single-layer and double-layer) and the cost of the extra sutures. Sta-

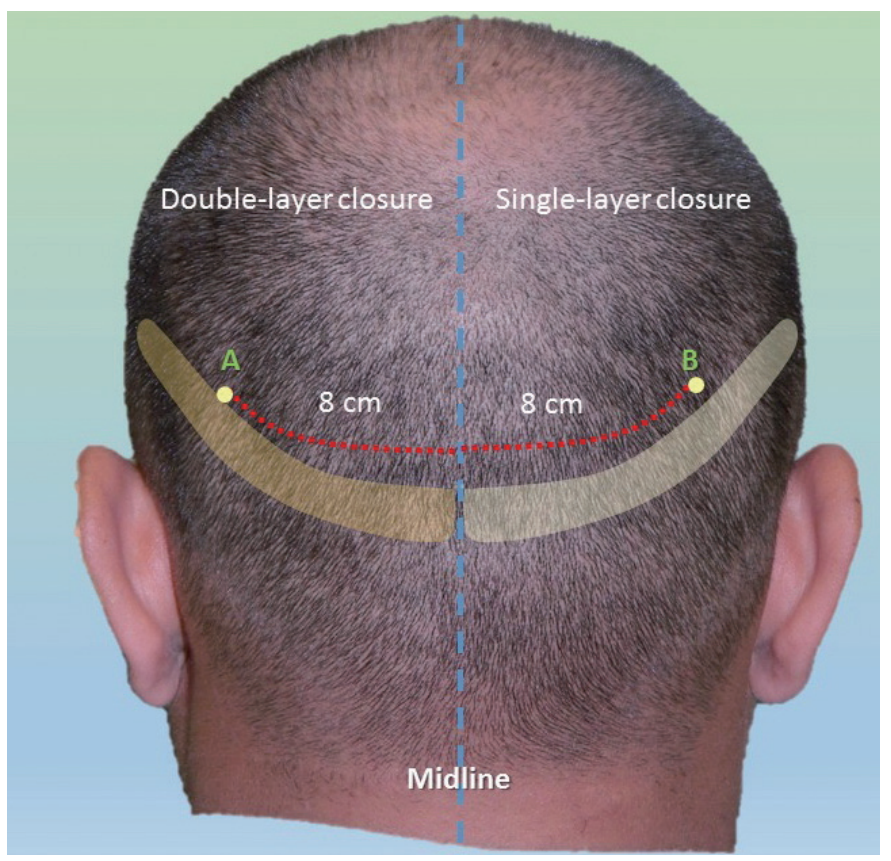

Fig. 1. Surgical protocol.

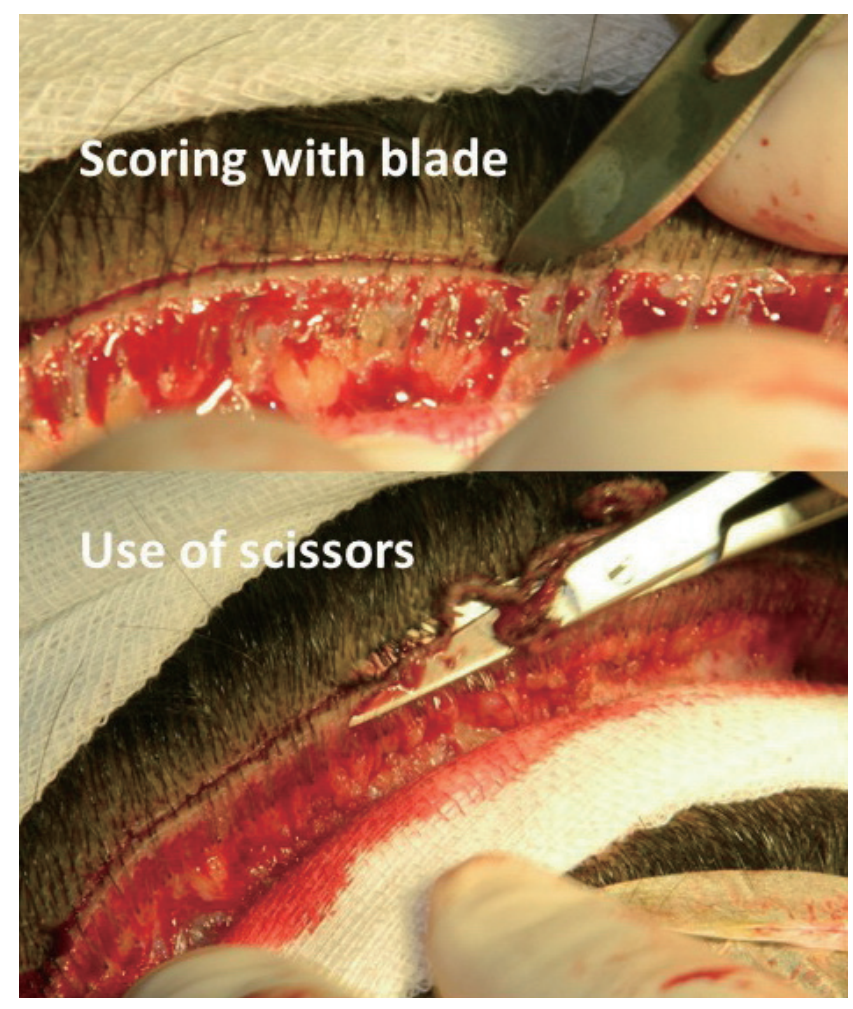

Fig. 2. Trichophytic wound closure.

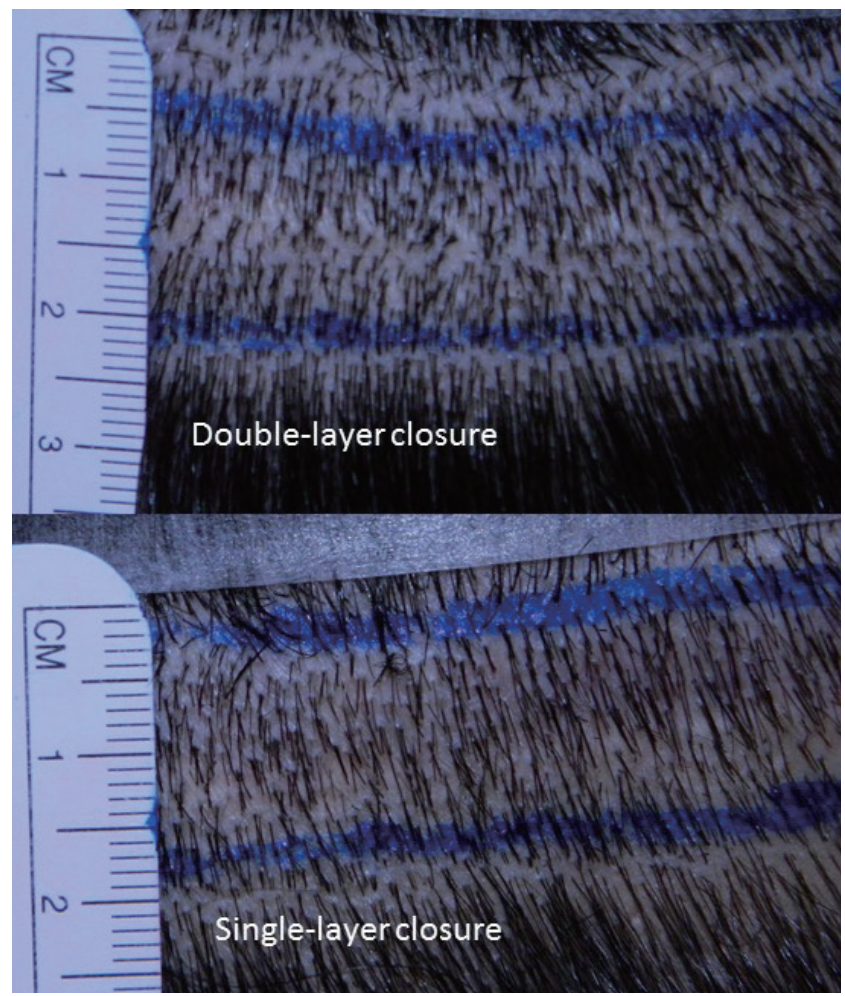

Fig. 3. Comparison of scars by single-layer vs. double-layer closure. 
Table 1. Patients' data

\begin{tabular}{|c|c|c|c|c|c|c|c|c|c|c|}
\hline \multirow{3}{*}{ Case no. } & \multirow{3}{*}{$\begin{array}{c}\text { Age } \\
\text { (year) }\end{array}$} & \multirow{3}{*}{ Type } & \multirow{3}{*}{$\begin{array}{l}\text { Width of } \\
\text { strip } \\
\text { (mm) }\end{array}$} & \multicolumn{2}{|c|}{ Scar width (mm) } & \multicolumn{2}{|c|}{ Mobility (mm) } & \multicolumn{3}{|c|}{ Time taken in closure (second) } \\
\hline & & & & \multirow{2}{*}{ 1-layer } & \multirow{2}{*}{ 2-layer } & \multirow{2}{*}{ 1-layer } & \multirow{2}{*}{ 2-layer } & \multirow{2}{*}{ 1-layer } & \multicolumn{2}{|c|}{ 2-layer } \\
\hline & & & & & & & & & Deep & Skin \\
\hline 1 & 29 & III & 19 & 1.9 & 2.0 & 5 & 4 & 205 & 45 & 200 \\
\hline 2 & 31 & IV & 18 & 1.6 & 1.5 & 5 & 3 & 220 & 51 & 225 \\
\hline 3 & 32 & IV & 15 & 1.3 & 1.3 & 4 & 4 & 220 & 59 & 215 \\
\hline 4 & 34 & V & 14 & 1.4 & 1.3 & 8 & 6 & 230 & 64 & 232 \\
\hline 5 & 39 & III & 13 & 1.0 & 1.0 & 13 & 9 & 232 & 62 & 234 \\
\hline 6 & 30 & IV & 14 & 1.1 & 1.2 & 12 & 11 & 239 & 54 & 229 \\
\hline 7 & 28 & III & 16 & 1.8 & 1.6 & 10 & 9 & 240 & 45 & 229 \\
\hline 8 & 46 & VII & 15 & 1.6 & 1.7 & 7 & 5 & 224 & 43 & 230 \\
\hline 9 & 35 & V & 13 & 1.9 & 1.8 & 8 & 6 & 215 & 54 & 229 \\
\hline 10 & 34 & V & 14 & 2.1 & 2.4 & 7 & 5 & 211 & 64 & 234 \\
\hline
\end{tabular}

tistical significance was calculated using Mann-Whitney U-test.

\section{RESULTS}

The mean age of the patients was 33.8 years (range, 28-46). The baldness pattern varied from type III to VII Norwood-Hamilton scale. The average width of the strip removed was $15.1 \mathrm{~mm}$ (range, 13-19 mm). The average width of the scar on single-layer closure was $1.57 \mathrm{~mm}$ and $1.58 \mathrm{~mm}$ on the double-layer closure (Fig. 3). The mobility of the scar was $7.9 \mathrm{~mm}$ on single-layer closure and $6.2 \mathrm{~mm}$ on double-layer closure (Table 1). The total time taken in single-layer closure was 223.6 seconds (3.727 minutes) as compare to double layer closure which was 279.8 seconds (4.663 minutes). The extra cost was about Pakistani Rupees 450 (about US Dollar 4) on half side of the wound.

\section{DISCUSSION}

Scar is an important sequelae of a hair transplant procedure. The scar maturation may take minimum of 6 months. The width of the scar is directly proportionate to the width of the strip removed and tension of the wound margins. The final scar is dependent on the pull exerted by the margins which may also be increased with muscular pull. As the donor scar is in the direction of the pull of scalp muscles, the ultimate scar is believed to stretch postoperatively. There are multiple factors which predict the final outcome. The final scar is dependent on various factors which include like the location of scar, the amount of width of scalp removed, the tension on wound margins etc. There have been controversy over the effectiveness of single-layer vs. double-layer closure of the donor strip in hair restoration surgery. There are certain pros and cons of each closure technique. The double-layer closure reduces the tension on the wound margins and decreases the dead space. It can result in buried suture material and can cause hair follicles damage. The one-layer closure has high possibility of 'stitch marks'. If wider resection (more than $1.2 \mathrm{~cm}$ ) is done, the scar may stretch due to elastic characteristics of the scalp. The location of the scar is very important. Any scar below the level of external occipital protuberance \& superior nuchal line, is bound to stretch due to the muscular movements of neck muscles. Moreover, the anatomy above and below the level of external occipital protuberance and superior nuchal line is different [8]. Similarly, the trichophytic closure helps to decrease the width of a scar as the hair grow through the scat and cause illusion of a smaller scar [9].

The study by Mubashir et al. [10] found no significant difference in scalp laceration closure (sub-dermal Vicryl and skin closure with prolene or staples). However the average width of the scar with prolene closure was lesser than staples ( $1.28 \mathrm{~mm}$ vs. 1.35 respectively). Bernstein et al. also found lesser scar on suture closure side [11]. There is no study which compare the quality of scar in single-layer and double-layer closure in the patients undergoing hair restoration (strip surgery).

In the current study, there was no significant difference in scar width in two techniques. However a few observations were noted which could add to the evidence-based medicine, i.e., the mobility of the scar. The scar in double-layer closure side had about $20 \%$ lesser mobility as compared to the single-layer closure side. The other study by Israr and Stassen [12] compare scalp closure with staples, silk, prolene and Vicryl rapide, and found no difference in healing in all four groups. But the scar width, postoperative mobility etc. were not noted. The study conducted by Muthuvel et al. [13] in skin closure with staples only (single-layer), the average width of the scar was $1.82 \mathrm{~mm}$ (range, $1-4 \mathrm{~mm}$ ) which is $15.4 \%$ more than the average scar noted in the current series (i.e., $1.54 \mathrm{~mm}$ ) [13]. More importantly the maximum scar width was $1.9 \mathrm{~mm}$ in the current study. 
The main aim of double-layer closure is to decrease the scar. It is thought that the deeper stitches hold the scar long enough till the forces from sides start pulling the scar, resulting in stretch. The normal wound healing has three main stages, inflammatory, proliferative and remodeling [14]. The first two phases last for about 3 weeks and at 3 weeks, the remodeling phase starts. At this time wound has only $20 \%$ of the tensile strength of the normal skin. The final scar has only 70 to $80 \%$ of the tensile strength at the end of $3 \mathrm{rd}$ phase [15]. Any foreign body/debris will slow down the wound healing by making body to work more. The life of Vicryl is about 2 week which may be decreased in vivo [16]. A study by Willat et al. [17], concluded that monofilamentous sutures (like Prolene, Polydioxanone) were far superior to catgut and silk. Another study by Agbakwuru et al. [18] found that there was no significant difference in healing of wound and appearance of scars in the patients having single versus two later closure after inguinal hernia repair. The average time taken in double-layer closure was more than the singlelayer closure (3.7 minutes vs. 4.7 minutes). However, the time was saved in single-layer wound closure $[18,19]$.

The final quality of scar is dependent on the amount of width of the strip which is removed during the surgery. Thus preoperative scalp mobility is very important point. There are various methods of assessing the scalp mobility but the Mayer's method is commonly used in which the compressibility is checked in horizontal direction. The method has a limitation as the donor wound closure is performed in vertical direction [20]. The vertical scalp mobility scale provides the opportunity to change the width of the strip according to the mobility in different areas [21].

The duration in single-layer vs double-layer closure was not noted in the current study but Adeolu et al. [22] found the single-layer closure to be much faster and cost-effective than double-layer closure. There is no scar assessment scale which could measure the quality of the scar especially in the cosmetic surgery. This also needs further studies to evaluate the amount of fibrosis and may be due to variation of body response to the foreign materials.

\section{CONCLUSION}

There is no difference in quality of scar in single layer vs. doublelayer closure. However, double-layer closure increased the overall cost and surgery time.

\section{PATIENT CONSENT}

Patients provided written consent for the use of their images.

\section{REFERENCES}

1. Gundlach KK, Maus C. Epidemiological studies on the frequency of clefts in Europe and world-wide. J Craniomaxillofac Surg 2006;34 Sup- pl 2:1-2.

2. Frans FA, van Zuijlen PP, Griot JP, et al. Assessment of scar quality after cleft lip closure. Cleft Palate Craniofac J 2012;49:171-6.

3. International Society of Hair Restoration Surgery. International society of hair restoration surgery: 2015 practice cdensus results. Geneva, IL: International Society of Hair Restoration Surgery, 2015. [cited by 2016 Jan 2]. Available from: http://www.ishrs.org/sites/default/files/ users/user3/report_2015_practice_census-final.pdf

4. Marzola M. Trichophytic closure of the donor area. Hair Transplant Forum Int 2005;15:113, 116.

5. Vogel JE, Jimenez F, Cole J, et al. Hair restoration surgery: the state of the art. Aesthet Surg J 2013;33:128-51.

6. Bernstein RM, Rassman WR. Staples revisited: Surgical staples in hair transplantation. Hair Transplant Forum Int 2008;18:10-1.

7. Ozturk D, Sonmez BM, Altinbilek E, et al. A retrospective observational study comparing hair apposition technique, suturing and stapling for scalp lacerations. World J Emerg Surg 2013;8:27.

8. Seery GE. Surgical anatomy of the scalp. Dermatol Surg 2002;28:581-7.

9. Frechet P. Minimal scars for scalp surgery. Dermatol Surg 2007;33:4555.

10. Mubashir S, Sajad P, Rasool F, et al. Conventional suture material versus staplers in the closure of donor area in hair transplant. Which is better? Hair Ther Transplant 2015;5:1000139.

11. Bernstein RM, Rassman WR, Rashid N. A new suture for hair transplantation: poliglecaprone 25. Dermatol Surg 2001;27:5-11.

12. Israr M, Stassen LF. The comparison of scalp closure with staples, silk, prolene and vicryl following a Gille's temporal approach for malar/ zygomatic complex fracture; a prospective study. Pak Oral Dent J 2013; 33:3-7.

13. Muthuvel K, Lakshmikanthan S, Subburathinam D. Outcomes of staple closure of the donor area during hair transplant by follicular unit transfer. J Cutan Aesthet Surg 2014;7:103-6.

14. Wasserbauer S, Creek W. Wound healing for the hair transplant surgeon. Hair Transplant Forum Int 2012;22:37, 42-4.

15. Gurtner GC. Wound healing: normal and abnormal. In: Thorne $\mathrm{CH}$, Bartlett SP, Beasley RW, et al., editors. Grabb and Smith's plastic surgery. 6th ed. Philadelphia, PA: Lippincott Williams \& Wilkins; 2006. p.15-22.

16. Bourne RB, Bitar H, Andreae PR, et al. In-vivo comparison of four absorbable sutures: Vicryl, dexon plus, maxon and PDS. Can J Surg 1988; 31:43-5.

17. Willatt DJ, Durham L, Ramadan MF, et al. A prospective randomized trial of suture material in aural wound closure. J Laryngol Otol 1988; 102:788-90.

18. Agbakwuru EA, Olabanji JK, Alatise OI, et al. Single versus two layer suturing for wound closure after inguinal hernia repair. Niger J Clin Pract 2009;12:162-4.

19. Moras K, Bhat M, Lasrado S, et al. Postaural incision closure in single layer versus multiple layers: a comparison. Indian J Otol 2014;20:60-2.

20. Mayer ML, Pauls T. Scalp elasticity scale. Hair Transplant Forum Int 
2005;15:122-3.

21. Mohmand MH, Ahmad M. Pre-operative estimation of scalp mobility for strip harvesting. Hair Transplant Forum Int 2016;26:198-9.
22. Adeolu AA, Olabanji JK, Komolafe EO, et al. A prospective study of two methods of closing surgical scalp wounds. Br J Neurosurg 2012; 26:75-7. 